\title{
Induratio penis plastica - eine Standortbestimmung
}

\author{
I. Schroeder-Printzen, W. Weidner \\ Urologische Klinik und Poliklinik der Georg-August-Universität in Göttingen (Direktor: Prof. Dr. R.-H. Ringert)
}

Am 4.10.1991 wurde vom AKA der deutschen Urologen in der Urologischen Universitätsklinik Göttingen die Induratio penis plastica (IPP) sieben Jahre nach einem Symposium in der Bonner Universitätsklinik im Sinne einer Standortbestimmung erneut in den Mittelpunkt einer andrologischen Tagung gestellt. An dem Symposium nahmen über 200 Teilnehmer aus allen deutschen Bundesländern, Österreich und der Schweiz teil. 13 Referenten stellten ihre Beiträge vor. Das Symposium stand unter der Leitung von Prof.Dr.med. W. Weidner (Göttingen) und Prof.Dr.med. G. Ludwig (Frankfurt a. M./Höchst).

Das Programm gliederte sich in folgende Punkte: neue Aspekte der Morphologie und Ätiopathogenese, Standardisierung der Diagnostik sowie medikamentöse und operative Therapie.

\section{Morphologie/Ätiopathogenese}

Die IPP ist eine Erkrankung des älteren Mannes mit einer Häufung im 5. und 6. Dezenium. G. E. Schubert (Wuppertal) bestätigte diese epidemiologischen Daten. In fast allen Fällen finden sich die Veränderungen am Penisrücken überwiegend basisnah. Schubert unterscheidet morphologisch ein Frühstadium mit einer entzündlichen Reaktion zwischen Tunika albuginea und Schwellkörper. Es folgt eine ausgedehnte Fibroblastenproliferation in der inneren Schicht der Plaques. Davon müssen Spätstadien abgegrenzt werden, bei denen Schubert zeigen konnte, daß der Fibroblastengehalt auch hier über dem von Tunika albuginea Gewebe bei kongenitaler Penisdeviation (dieses diente als Kontrollmaterial) liegt. Dabei ist besonders interessant, da $\beta$ diese Veränderungen in der gesamten Tunika albuginea, nicht nur im Plaques, nachweisbar sind. Diese Befunde werfen erneut die Frage auf, ob die IPP nur als lokalisierte Erkrankung oder als fibrotische Manifestation einer generalisierten penilen Erkrankung zu werten ist. Kalzifizierungen im Penis sind selten. Darüber hinaus erscheint wichtig darauf hinzuweisen, daß auch nach längerer Erkrankungsdauer (bis zu 4 Jahren) perivaskuläre entzündliche Infiltrate in den Plaques nachzuweisen sind. Das Fortbestehen dieser Veränderungen kann den im Einzelfall schwer voraussagbaren Verlauf mit unterschiedlicher Progredienz oder auch Spontanremission (in der Literatur bis zu $50 \%$ ) eventuell erklären.
R. Rompel (Kassel, Gießen) erläuterte in seinem Vortrag die Einordnung der IPP in den Formenkreis der Fibromatosen. Er berichtete über eine entsprechende Assoziation mit der Dupuytrenschen Kontraktur und Plantarfibrose (Morbus Ledderhose) sowie Fingerknöchelpolstern. Immungenetische Untersuchungen der Gießener Arbeitsgruppe haben gezeigt, daß entsprechende Vergesellschaftungen eventuell durch Assoziation von HLA-Antigenen erklärt werden können, wobei insbesondere eine signifikante HLA-Assoziation für HLA-DR3 und HLA-DQw2 im Vergleich mit gesunden Kontrollpersonen (Blutspendern) nachgewiesen worden ist. Weitere prospektive Untersuchungen sind anzustreben, um eine derartig lange vermutete immunologische Disposition der IPP $\mathrm{zu}$ verifizieren.

\section{Diagnostik}

Die Diagnostik wurde von $W$. Weidner (Göttingen), G. Helweg (Innsbruck) und in der Diskussion von G. Ludwig (Frankfurt), V. Barth (Aue), sowie J. Schubert (Jena) ausführlich erörtert. Aufgrund der Erfahrungen in der Gießener und Göttinger Andrologischen Sprechstunde bestätigte Weidner die häufige Assoziation mit den bereits oben beschriebenen Fibromatosen vom Dupuytren-Typ. In einer Serie von 52 konsekutiven unbehandelten Patienten mit genuiner IPP fand er immerhin in $30,8 \%$ eine vergesellschaftete Dupuytrenschen Kontraktur, in $23,1 \%$ Fingerknöchelpolster und in 2,8\% einen Morbus Ledderhose.

Besonders interessant ist für die Diagnostik die Häufigkeit einer Erektionsstörung, die nach ersten amerikanischen Untersuchungen in bis $\mathrm{zu} 62 \%$ aller $\mathrm{Pa}$ tienten mit IPP aufgrund plaques-assoziierter pathologischer venöser Abflüsse auftreten soll. Weidner fand in seiner Serie eine manifeste Erektionsstörung nur bei $8 / 52$ Männern, wobei in der Diskussion jedoch eindeutig herausgearbeitet wurde, daß die Selektion des Patientengutes und insbesondere die Frage der Vorbehandlung eine entscheidende Rolle für die Inzidenz dieser Veränderungen spielt. Ausführlich diskutiert wurde in diesem Zusammenhang auch die Wertigkeit eines routinemäßigen intrakavernösen Pharmakontestes bei Patienten mit IPP. Konsens bestand insoweit, da $\beta$ die meisten der sich mit IPP befassenden Kollegen eine Pharmakonaustestung zur Verifizierung einer Erektionsstörung unter Einschluß einer Dopplersonographie, einer penilen Sonographie und gleichzeitigen objektiven Bestimmung des Deviationswinkels des Penis für angebracht halten (J. Schubert, Jena).

Der Definition der Plaques mit bildgebenden Verfahren hat sich insbesondere die Arbeitsgruppe 
von Barth (Aue) angenommen. Er beschäftigt sich mit Ultraschallbefunden im Bereich der Plaques unter Verwendung der Duplexsonographie, wobei neben der Größenbestimmung eine Messung der Gefäßdurchmesser der Schwellkörperarterien und ihre Strömungsgeschwindigkeit nach Injektion von vasoaktiven Substanzen als sinnvoll bei der IPP angegeben wird. Weidner führt nur eine Schwellkörpersonographie in 2 Ebenen zum Nachweis eines verkalkenden Herdes (Schlagschatten) durch und meint anhand seiner Untersuchungen nachgewiesen $\mathrm{zu}$ haben, da $\beta$ die penile Sonographie mit entsprechender Wasservorlaufstrecke durch Nachweis einer hyperreflexiven Plaqueszone in diesen Fällen durchaus zur Klassifikation der Erkrankung beitragen kann. Die Diagnostik der Wahl bei Verdacht auf zusätzliche entzündliche oder ödematöse Veränderungen scheint jedoch die Kernspintomographie, insbesondere unter Einsatz von entsprechenden Kontrastmitteln (Gadolinium DTPA) und Anfertigung von $\mathrm{T}_{1}$-gewichteten schnellen Sequenzen (Helweg, Innsbruck) zu sein. Sie erreicht hier eine deutlich höhere Sensitivität. Sollten nur Plaques nachgewiesen werden, hat die Sonographie in der Hand des geübten Untersuchers deutliche Vorteile.

\section{Therapie}

G. Ludwig, W. Aulitzky (Salzburg) und G.W. Müller (Magdeburg) berichteten über die medikamentöse systemische Behandlung und lokale Injektionstherapie bei der IPP. Als Therapiestrategien der Wahl wurden dabei die orale Therapie mit Kalium-4-aminobenzoat (Potaba ${ }^{\circledR}$ ) und die Injektionstherapie mit Orgotein (Peroxinorm ${ }^{\circledR}$ ) diskutiert. Ludwig sieht aufgrund fehlender Wirksamkeit derzeit keinerlei Indikation für eine orale Medikamententherapie. Dagegen berichtet die Gruppe um Müller (Magdeburg) bei genuiner, nicht verkalkender IPP über ermutigende Erfolge mit Potaba ${ }^{\circledR}$ insbesondere zur Beseitigung des Erektionsschmerzes, der nach den Erfahrungen dieser Arbeitsgruppe in bis zu $100 \%$ kupiert wird. Aus der anschließenden zum Teil leidenschaftlich geführten Diskussion ergab sich eindeutig die Notwendigkeit, die Wirksamkeit einer oralen Medikation, z.B. von Kalium-4aminobenzoat im Sinne einer randomisierten prospektiven plazebokontrollierten Studie zu überprüfen. Der Arbeitskreis Andrologie der deutschen Urologen bereitet eine solche Studie derzeit vor. Durchaus widersprüchlich wurde auch die Injektionstherapie z. B. mit Orgotein diskutiert. Aulitzky berichtete über die positiven Erfahrungen seiner Arbeitsgruppe. In der Diskussion konnten jedoch die Bedenken wegen der berichteten allergischen Reaktionen (Häufigkeit, 1,4 auf 10000 Behandlungen) nicht ganz zerstreut werden.

Einen besonders interessanten Aspekt behandelte $H$. J. Vogt (München) mit seinem Bericht über die Röntgenweichstrahltherapie bei der IPP. Vogt behandelt mit dem Dermopan ${ }^{\circledR}$ mit einer Gesamtdosis von 3200 $\mathrm{R}$ und einer Einzeldosis von $400 \mathrm{R}$ an jeweils zwei aufeinanderfolgenden Tagen in achtwöchigen Abständen. Er kommt in seinen Untersuchungsergebnissen dabei, bezogen auf den Tastbefund, zu einer deutlichen Befundbesserung, wobei in vielen Fällen posttherapeutisch nur noch eine Restinduration nachgewiesen werden konnte. Der heftigen Kritik an dieser Therapieform, die sich in der
Diskussion insbesondere von operativer Seite daran entzündete, daß eventuelle plastische Maßnahmen am Penis nach dieser Therapie nicht mehr möglich sind bzw. deutlich erschwert würden, wurde jedoch auch von urologischer Seite widersprochen. Nach unserer Meinung limitiert sich dieser Therapieansatz dadurch, daß das Dermo$\operatorname{pan}^{\circledR}$-Gerät nur noch in den wenigsten Hautkliniken zur Röntgenweichstrahltherapie zur Verfügung steht.

In einem interessanten Kurzvortrag berichtete V. Maso (Frankfurt/Moskau) über seine Erfahrung mit der Softlaserbehandlung bei mehreren hundert Patienten, wobei er diese als besonders effektiv und nebenwirkungsfrei darstellte. Die Autoren können den Erfolg dieses Therapieansatzes, der auch bereits von anderer Seite in bis zu $43,3 \%$ beschrieben worden ist, nicht eindeutig einordnen und glauben, $\mathrm{da} \beta$ weitere prospektive Untersuchungen notwendig sind, um definitiv zu diesem Verfahren Stellung zu nehmen.

Zur operativen Therapie berichteten $F$. Schreiter (Schwelm), J. Schubert (Jena) und B. Ulshöfer (Marburg) über ihre Erfahrungen der letzten Jahre. Übereinstimmend wurde festgestellt, daß bei IPP mit Penisdeviation und/oder Verkalkung unbedingt eine operative Korrektur notwendig ist, da eine medikamentöse Therapie nicht zu einer ausreichenden Rückbildung in diesem Stadium der Erkrankung führt. Als Verfahren wurden die plaquesschonenden kontralateralen Korporoplastiken wie Nesbit-Operation und Plikation sowie Plaquesresektion mit Deckung mit und ohne Implantation einer Penisprothese diskutiert. J. Schubert (Jena) analysierte seine Erfahrungen mit der Plikationstechnik an 54 Patienten. In seiner Hand ist diese Therapie bei Verwendung von nicht resorbierbarem Material komplikationslos bei gleichzeitigem Verzicht auf eine Eröffnung des Schwellkörpers und Resektion der Plaques. Nachteile sieht seine Arbeitsgruppe in Übereinstimmung mit Schreiter und anderem in einer bis zu $3 \mathrm{~cm}$ Länge möglichen Verkürzung des Penis. Uns erscheint die Plikatur beim erektionsfähigen Patienten mit Deviation das Therapieverfahren der Wahl zu sein. Entsprechende positive Erfahrungen wurden auch von Ulshöfer dargestellt.

Schreiter berichtete über sein operatives Behandlungsschema bei der IPP. Dabei favorisiert er die Korporoplastik mit und ohne Exzision der Tunika albuginea, wobei er die Implantation einer Penisprothese allein zur Korrektur einer Schaftverkrümmung ablehnt. Das Nesbitsche Verfahren führt er analog zu Schubert (Jena) bei Patienten mit Deviation ohne Erektionsstörung durch. Grenzen dieser Technik sieht Schreiter insbesondere bei kleinem Penis und auch bei Rotationsverkrümmungen oder zirkulärer Einengung. In diesen Fällen wird die Plaquesexzision mit anschließender Deckung des Tunika albuginea-Defektes im Sinne einer "Dermal Graft" Corporoplastik nach Horton und Devine durchgeführt. Schreiter empfiehlt hierzu die Verwendung einer Lupenbrille. Bei vorhandenem Erektionsverlust bevorzugt Schreiter die Implantation einer hydraulischen Prothese, kombiniert mit einer Plaqueexzision und Deckung des Tunikadefektes mit alloplastischem Material, insbesondere Goretex oder Dacron. Nach allgemeiner Übereinstimmung sollte jedoch die Implantation einer Penisprothese von einer begleiten- 
den erektilen Dysfunktion abhängig gemacht werden, die ja in vielen Fällen cavernosographisch und cavernosometrisch „plaques-assoziiert“ nachgewiesen werden kann. Sicher erscheint, daß eine Dermal Graft-Plastik nach dem heutigen Wissensstand nur bei voll erhaltener erektiler Funktion angestrebt werden sollte. Tritt dennoch postoperativ eine sekundäre erektile Dysfunktion auf, wird eine hydraulische Prothese implantiert.

Zusammenfassend konnte auf der Arbeitstagung nur in Teilaspekten ein allgemein akzeptiertes Konzept zur Therapie der IPP erarbeitet werden. Einigkeit bestand, daß in den meisten Fällen bei nicht kalzifizierendem Herd und dem Leitsymptom Erektionsschmerz der Versuch einer primären konservativen Therapie zu diskutieren ist. Um so wichtiger erscheint eine prospektive Studie, die die Effektivität eines konservativen Therapieansatzes bestätigt oder endgültig widerlegt. Bei Deviation ist eine Begradigung durch Nesbit-Op bzw. Plikation nur dann sinnvoll, wenn kein Erektionsverlust besteht. Bei gravierenden Fällen, Verkalkungen etc., muß auch hier eine Exzision der Plaques bei gleichzeitiger alloplastischer Deckung diskutiert werden. Unumgänglich bei Erektionsstörungen ist sicher die (zusätzliche) Implantation einer Penisprothese nach entsprechender Exzision und Dekkung des Resektionsbereiches.

\section{Ausblick}

Langzeitverläufe der bisher operativ versorgten Patienten und die Weiterführung der ätiologischen und immunologischen Untersuchungen bei Patienten mit IPP werden zeigen, ob dieses in weiten Bereichen noch wenig bekannte Krankheitsbild derzeit von uns richtig diagnostisch und therapeutisch angegangen wird.
Dr.med. I. Schroeder-Printzen

Urologische Klinik und Poliklinik der Georg-August-Universität Robert-Koch-Str. 40

3400 Göttingen 